\title{
A local Jacobian smoothing method for solving Nonlinear Complementarity Problems
}

\author{
Favián Arenas ${ }^{1}$, Héctor Jairo Martínez ${ }^{2}$, Rosana Pérez ${ }^{1, *}$
}

\section{Edited by}

Juan Carlos Salcedo-Reyes

(salcedo.juan@javeriana.edu.co)

1. Universidad del Cauca,

Popayán - Colombia

2. Universidad del Valle

Cali - Colombia.

*rosana@unicauca.edu.co

Received: 13-11-2018

Accepted: 21-01-2019

Published on line: 01-05-2020

Citation: Arenas F, Martínez HJ,

Pérez R. A local Jacobian smoothing

method for solving Nonlinear

Complementarity Problems,

Universitas Scientiarum, 25 (1): 149-174, 2020.

doi: 10.11144/Javeriana.SC25-1.aljs

\section{Funding:}

Universidad del Cauca Research Project

VRI ID 4511.

Electronic supplementary material: N.A.

OPEN ACCESS

\begin{abstract}
In this paper, we present a smoothing of a family of nonlinear complementarity functions and use its properties in combination with the smooth Jacobian strategy to present a new generalized Newton-type algorithm to solve a nonsmooth system of equations equivalent to the Nonlinear Complementarity Problem. In addition, we prove that the algorithm converges locally and $q$-quadratically, and analyze its numerical performance.
\end{abstract}

Keywords: nonlinear complementarity problem; complementarity function; generalized Newton method; Q-quadratic convergence.

\section{Introduction}

Let $F: \mathbb{R}^{n} \rightarrow \mathbb{R}^{n}$ be a continuously differentiable function. The Nonlinear Complementarity Problem, NCP for short, is to find a vector $x \in \mathbb{R}^{n}$ satisfying the following conditions

$$
x \geq 0, \quad F(x) \geq 0, \quad x^{T} F(x)=0 .
$$

We say that a vector in $\mathbb{R}^{n}$ is nonnegative if each of its components is nonnegative.

There are numerous and varied applications of the NCP in Engineering [1, 2] and Economics $[3,4]$. In this last area, complementarity and economic equilibrium are synonymous.

One technique, perhaps the most popular, to solve nonlinear complementarity problems is to write them in an equivalent way as a nonlinear system of equations. In this process, it is used a function $\varphi: \mathbb{R}^{2} \rightarrow \mathbb{R}$ such that 


$$
\varphi(a, b)=0 \Longleftrightarrow a \geq 0, b \geq 0, a b=0,
$$

called a complementarity function [5].

The equivalence (1) allows to conclude that a complementarity function is nondifferentiable due to the lack of smoothness of its trace by the intersection with the $x y$ plane which is not differentiable at $(0,0)$.

Given a complementarity function $\varphi$ and a function $\Phi: \mathbb{R}^{n} \rightarrow \mathbb{R}^{n}$, we define the nonlinear system of equations by

$$
\Phi(x)=\left(\begin{array}{c}
\varphi\left(x_{1}, F_{1}(x)\right) \\
\vdots \\
\varphi\left(x_{n}, F_{n}(x)\right)
\end{array}\right)=0,
$$

which is nondifferentiable due to the lack of smoothness of $\varphi$. From (1), it follows that a necessary and sufficient condition for a vector $\boldsymbol{x}_{*}$ to solve the NCP is that this vector solves the system (2).

Two examples of complementarity functions, widely used, are the following

$$
\varphi(a, b)=\min \{a, b\}, \quad \varphi(a, b)=\sqrt{a^{2}+b^{2}}-a-b,
$$

which are called the Minimum function [6] and the Fischer-Burmeister function [7], respectively.

Other example of complementarity functions is the family $\varphi_{\lambda}$ introduced in [7] and defined by

$$
\varphi_{\lambda}(a, b)=\sqrt{(a-b)^{2}+\lambda a b}-a-b,
$$

where $\lambda \in(0,4)$.

It is important to observe that the Minimum function [6] and the FischerBurmeister function are particular cases of the family $\varphi_{\lambda}$.

The nonsmooth system of nonlinear equations (2), equivalently the NCP, has been solved using nonsmooth methods of Nerwton [8] and quasi-Nerwton $[9,10,11,12]$ type, and smooth methods $[13,14,15]$. These methods are based on Clarke's generalized Jacobian [16] defined by a Lipschitz continuous function $G: \mathbb{R}^{n} \rightarrow \mathbb{R}^{n}$ as follows

$$
\partial G(x)=\operatorname{hull}\left\{\lim _{k \rightarrow \infty} F^{\prime}\left(x_{k}\right) \in \mathbb{R}^{n \times n}: x_{k} \rightarrow x, x_{k} \in D_{G}\right\},
$$

where $D_{G}$ denotes the set of all points where $G$ is differentiable and hull $(A)$ is the convex envelope of $A$. In general, this set is difficult to compute [17]; in this paper, we use the overestimation given by [18, Proposition 2.6.2 (e)],

$$
\partial G(\boldsymbol{x})^{T} \subseteq \partial G_{1}(\boldsymbol{x}) \times \ldots \times \partial G_{n}(\boldsymbol{x}) \equiv \partial_{C} G(\boldsymbol{x})^{T},
$$


where the right-hand side (often easier to compute [19]) denotes the set of matrices in $\mathbb{R}^{n \times n}$ whose $i$-th column is given by the generalized gradient of the $i$-th component function $G_{i}$. The set $\partial_{C} G(x)$ is called the $C$-subdifferential of $G$ at $\boldsymbol{x}$.

The nonsmooth Newton methods $[8,19]$ solve at each iteration the generalized Nerton equation

$$
H_{k} s_{k}=-\Phi\left(x_{k}\right)
$$

where $H_{k} \in \partial \Phi\left(\boldsymbol{x}_{k}\right)$, or $H_{k} \in \partial_{C} \Phi\left(\boldsymbol{x}_{k}\right)$.

A way to deal with the nonsmoothness of $\Phi$ and to solve (2) is to use a Jacobian smoothing method introduced in [20]. The basic idea of this type of methods is to approximate $\Phi$ by a smooth operator $\Phi_{\mu}: \mathbb{R}^{n} \rightarrow \mathbb{R}^{n}$, where $\mu>0$ denotes the smoothing parameter, and then to solve a sequence of problems

$$
\Phi_{\mu}(\boldsymbol{x})=0
$$

forcing $\mu$ to go to zero. For this, a Jacobian smoothing method tries to solve at each iteration the mixed Newton equation

$$
\Phi_{\mu}^{\prime}\left(\boldsymbol{x}_{k}\right) s_{k}=-\Phi\left(\boldsymbol{x}_{k}\right)
$$

where $\Phi_{\mu}^{\prime}\left(\boldsymbol{x}_{k}\right)$ is the Jacobian matrix of the function $\Phi_{\mu}$ at $\boldsymbol{x}_{k}$. The linear system (7) uses the unperturbed right-hand side of equation (5), but it replaces the $H_{k} \in \partial \Phi_{\lambda}\left(\boldsymbol{x}_{k}\right)$ by a suitable approximation $\Phi_{\mu}^{\prime}\left(\boldsymbol{x}_{k}\right)$.

The authors in [20] developed the convergence theory of Jacobian smoothing methods for a special type of functions. A new algorithm for general functions was presented in [17], where the authors use the strategy of JAcobian smoothing to solve the NCP by reformulating it as a system of non-linear equations using the Fischer-Burmeister complementarity function.

The good results obtained in [17] and the fact that the family of functions (3) has not been used in connection with the Jacobian smoothing method motivated us to use that strategy to solve the NCP through its reformulation as a non-differentiable non-linear system, using a one-parameter family of complementarity functions (3), which we have analyzed and used recently in $[21,22]$. That is, we consider the nonsmooth nonlinear system of equations

$$
\Phi_{\lambda}(x)=\left(\begin{array}{c}
\varphi_{\lambda}\left(x_{1}, F_{1}(x)\right) \\
\vdots \\
\varphi_{\lambda}\left(x_{n}, F_{n}(x)\right)
\end{array}\right)=0 .
$$


The function $\Phi_{\lambda}$ is locally Lipschitz continuous because of the Lipschitz continuity of $\varphi_{\lambda}$ (see [23]). Therefore, $\partial \Phi_{\lambda}(\boldsymbol{x})$ exists.

In this paper, we propose and analyze a smoothing of the family of nonlinear complementarity functions proposed in [7] and we use its properties in combination with the smooth Jacobian strategy used in [17] to present a new generalized Newton-type algorithm to solve a nonsmooth system of equations equivalent to the Nonlinear Complementarity Problem. In addition, we prove that the algorithm converges locally and $q$-quadratically, analyzing also its numerical performance.

The organization of this paper is as follows: In Section 2, we present a smoothing of a one-parameter family of complementarity functions (3), and analyze its properties. In Section 3, we present a new algorithm that uses the Jacobian smoothing strategy and develop its local convergence theory. In Section 4, we present numerical experiments which permit us to analyze the performance of the proposed algorithm. Finally, in Section 5, we present our concluding remarks.

\section{Smoothing a family of complementarity functions}

In this section, we define and analyze a smooth approximation of the family of complementarity functions (3) introduced in [7], which was redefined and analyzed in detail in [23]. Following this reference, we use the $G_{\lambda}(a, b)$ notation for the first term on the right side of (3). That is, $G_{\lambda}(a, b)=$ $\sqrt{(a-b)^{2}+\lambda a b}$.

Definition 2.1. The function $\varphi_{\lambda \mu}$ given by

$$
\varphi_{\lambda \mu}(a, b)=\sqrt{(a-b)^{2}+\lambda a b+(4-\lambda) \mu}-a-b, \lambda \in(0,4), \mu>0
$$

is a smooth approximation of a family of complementarity functions $\varphi_{\lambda}$.

The function $\varphi_{\lambda \mu}$ is well defined for all $\lambda \in(0,4)$ and for all $\mu>0$. Indeed,

$$
(a-b)^{2}+\lambda a b+(4-\lambda) \mu=\left(\begin{array}{ll}
a & b
\end{array}\right) K\left(\begin{array}{l}
a \\
b
\end{array}\right)+(4-\lambda) \mu>0,
$$

where

$$
K=\frac{1}{2}\left(\begin{array}{cc}
2 & \lambda-2 \\
\lambda-2 & 2
\end{array}\right)
$$

is a symmetric and positive definite matrix [23].

The following lemma guarantees that $\varphi_{\lambda \mu}$ is a "perturbed" complementarity function. 
Lemma 2.2. The function $\varphi_{\lambda \mu}$ satisfies the following equivalence

$$
\varphi_{\lambda \mu}(a, b)=0 \Longleftrightarrow a \geq 0, b \geq 0, a b=\mu .
$$

Proof. It is a direct consequence of the definition of $\varphi_{\lambda \mu}$.

A useful bound for later theoretical developments is given in the following lemma.

Lemma 2.3. Lets $\lambda \in(0,4)$ and $\mu>0$. The function $G_{\lambda \mu}$, defined for all $(a, b) \in \mathbb{R}^{2}$ by

$$
G_{\lambda \mu}(a, b)=\sqrt{(a-b)^{2}+\lambda a b+(4-\lambda) \mu},
$$

satisfies the following inequality

$$
G_{\lambda \mu}(a, b) \geq \sqrt{\alpha_{\min }}\left\|\left(\begin{array}{l}
a \\
b
\end{array}\right)\right\|_{2},
$$

where $\alpha_{\min }>0$ is the smallest eigenvalue of the matrix $K$ defined by (10).

Proof. Let $\alpha_{\min }>0$ be the smallest eigenvalue of the matrix $K$ in (10), then

$$
\begin{aligned}
G_{\lambda \mu}(a, b) & =\sqrt{(a-b)^{2}+\lambda a b+(4-\lambda) \mu} \geq \sqrt{(a-b)^{2}+\lambda a b} \\
& \geq \sqrt{\alpha_{\min }}\left\|\left(\begin{array}{l}
a \\
b
\end{array}\right)\right\|_{2},
\end{aligned}
$$

where the last inequality is given by [23, Lemma 1$]$.

Observe that the function $\varphi_{\lambda}$ is nondifferentiable at $(0,0)$ but its smoothing function $\varphi_{\lambda \mu}$ is differentiable, and its gradient vector is defined by

$$
\nabla \varphi_{\lambda \mu}(a, b)=\left(\begin{array}{l}
\frac{2(a-b)+\lambda b}{2 G_{\lambda \mu}(a, b)}-1 \\
\frac{-2(a-b)+\lambda a}{2 G_{\lambda \mu}(a, b)}-1
\end{array}\right)=\nabla G_{\lambda \mu}(a, b)-\left(\begin{array}{l}
1 \\
1
\end{array}\right) .
$$

For later use, we will denote the partial derivatives of $G_{\lambda \mu}$ as follows

$$
\alpha_{\lambda \mu}(a, b)=\frac{2(a-b)+\lambda b}{2 G_{\lambda \mu}(a, b)}, \quad \text { and } \quad \beta_{\lambda \mu}(a, b)=\frac{-2(a-b)+\lambda a}{2 G_{\lambda \mu}(a, b)},
$$

and the derivatives of $G_{\lambda}$ by

$$
\alpha_{\lambda}(a, b)=\frac{2(a-b)+\lambda b}{2 G_{\lambda}(a, b)}, \quad \text { and } \quad \beta_{\lambda}(a, b)=\frac{-2(a-b)+\lambda a}{2 G_{\lambda}(a, b)} .
$$


From $G_{\lambda \mu}(a, b) \geq G_{\lambda}(a, b)$ and $[\underline{7}$, Lemma 2.4], we have

$$
\left\|\nabla G_{\lambda \mu}(a, b)\right\|_{2} \leq \sqrt{2}
$$

From (13), (16) and the triangle inequality, we have

$$
\left\|\nabla \varphi_{\lambda \mu}(a, b)\right\|_{2} \leq 2 \sqrt{2}
$$

Following [23], we have a matrix expression for $\nabla G_{\lambda \mu}(a, b)$ analogous to the one given for $\nabla G_{\lambda}(a, b)$, namely

$$
\nabla G_{\lambda \mu}(a, b)=\frac{1}{G_{\lambda \mu}(a, b)} K\left(\begin{array}{l}
a \\
b
\end{array}\right),
$$

where $K$ is the matrix given by (10) which satisfies $\|K\|_{2}<2$.

An important property of the smoothing function $\varphi_{\lambda \mu}$ is that it is an uniformly continuous function; moreover, it is Lipschitz continuous as guaranteed by the following lemma.

Lemma 2.4. The function $\varphi_{\lambda \mu}$ is Lipschitz continuous with constant $2 \sqrt{2}$. That is, for all $x, y \in \mathbb{R}^{2}$

$$
\left|\varphi_{\lambda \mu}(x)-\varphi_{\lambda \mu}(y)\right| \leq 2 \sqrt{2}\|x-y\|_{2} .
$$

Proof. Let $x, y \in \mathbb{R}^{2}$. By the Mean Value Theorem, there exists a vector $z$ in $(x, y)$ such that,

$$
\varphi_{\lambda \mu}(x)-\varphi_{\lambda \mu}(y)=\nabla \varphi_{\lambda \mu}(z)^{T}(x-y)
$$

using a Cauchy-Schwartz inequality and the bound (17), we have

$$
\left|\varphi_{\lambda \mu}(x)-\varphi_{\lambda \mu}(y)\right| \leq\left\|\nabla \varphi_{\lambda \mu}(z)\right\|_{2}\|x-y\|_{2} \leq 2 \sqrt{2}\|x-y\|_{2}
$$

thus, we conclude that $\varphi_{\lambda \mu}$ is Lipschitz continuous with constant $2 \sqrt{2}$.

Corollary 2.5. The function $G_{\lambda \mu}$ is Lipschitz continuous, that is, for all possible $x, y \in \mathbb{R}^{2}$, we have the following bound

$$
\left|G_{\lambda \mu}(x)-G_{\lambda \mu}(y)\right| \leq \sqrt{2}\|x-y\|_{2} .
$$

Proof. It is analogous to the proof of the previous lemma.

The following lemma guarantees that $\nabla \varphi_{\lambda \mu}$ is also a locally Lipschitz continuous function. 
Lemma 2.6. Let $\varphi_{\lambda \mu}$ the function defined by (9), w a nonzero vector in $\mathbb{R}^{2}$ and $\mathscr{B}(w ; \epsilon)$ a ball with $0<\epsilon<\frac{1}{2}\|w\|_{2}$. Then there exists $\eta>0$ such that for all $u, v \in \mathscr{B}(w ; \epsilon)$,

$$
\left\|\nabla \varphi_{\lambda \mu}(u)-\nabla \varphi_{\lambda \mu}(v)\right\|_{2} \leq \eta\|u-v\|_{2} .
$$

Proof. It is similar to the proof of [23, Lemma 5] using (12), (16), (18), and the Corollary 2.5 .

Lemma 2.7. Let $\varphi_{\lambda}$ and $\varphi_{\lambda \mu}$ the functions defined by (3) and (9), respectively; $w$ a nonzero vector in $\mathbb{R}^{2}$ and $\mathscr{B}(w ; \epsilon)$, with $0<\epsilon<\frac{1}{2}\|w\|_{2}$, a ball that does not contain $(0,0)$. Then there exists $\tau>0$ such that, for all $u, v \in \mathscr{B}(w ; \epsilon)$,

$$
\left\|\nabla \varphi_{\lambda \mu}(u)-\nabla \varphi_{\lambda}(v)\right\|_{2} \leq \tau\|\boldsymbol{u}-v\|_{2} .
$$

Proof. It is analogous to the proof of [23, Lemma 5] taking into account the inequality $G_{\lambda}(c, d) \leq G_{\lambda \mu}(c, d)$ and therefore,

$$
\left|\frac{1}{G_{\lambda \mu}(a, b)}-\frac{1}{G_{\lambda}(c, d)}\right|=\left|\frac{G_{\lambda}(c, d)-G_{\lambda \mu}(a, b)}{G_{\lambda \mu}(a, b) G_{\lambda}(c, d)}\right| \leq\left|\frac{G_{\lambda \mu}(c, d)-G_{\lambda \mu}(a, b)}{G_{\lambda \mu}(a, b) G_{\lambda}(c, d)}\right| .
$$

\section{Algorithm and convergence theory}

Following the idea of Jacobian smoothing methods presented in the introduction of this paper, we propose the following basic algorithm for solving the nonsmooth nonlinear system of equations (8).

Algorithm 3.1. Given $x_{0}$, an initial point, $\lambda \in(0,4)$, and $\left\{\mu_{k}\right\}$ a sequence that converges to zero, for $k=1,2, \ldots$, compute

$$
\begin{aligned}
\Phi_{\lambda \mu}^{\prime}\left(\boldsymbol{x}_{k}\right) s_{k} & =-\Phi_{\lambda}\left(\boldsymbol{x}_{k}\right), \\
\boldsymbol{x}_{k+1} & =\boldsymbol{x}_{k}+\boldsymbol{s}_{k},
\end{aligned}
$$

where $\Phi_{\lambda \mu}^{\prime}\left(\boldsymbol{x}_{k}\right)$ is the Jacobian matrix of the function $\Phi_{\lambda \mu}$ at $\boldsymbol{x}_{k}$.

It is important to observe that we use the matrix $\Phi_{\lambda \mu}^{\prime}\left(\boldsymbol{x}_{k}\right)$ instead of $H_{k} \in$ $\partial_{C} \Phi_{\lambda}\left(x_{k}\right)$.

The first result characterizes the matrices of the C-subdifferential of $\Phi_{\lambda}$ at $\boldsymbol{x}$.

Proposition 3.2. For an arbitrary $x \in \mathbb{R}^{n}$, we have

$$
\partial_{C} G(\boldsymbol{x})^{T}=D_{\alpha}(\boldsymbol{x})+F^{\prime}(\boldsymbol{x})^{T} D_{\beta}(\boldsymbol{x})
$$


where $D_{\alpha}(\boldsymbol{x})=\operatorname{diag}\left(\alpha_{1}(\boldsymbol{x}), \ldots, \alpha_{n}(\boldsymbol{x})\right)$ and $D_{\beta}(\boldsymbol{x})=\operatorname{diag}\left(\beta_{1}(\boldsymbol{x}), \ldots, \beta_{n}(\boldsymbol{x})\right)$ are diagonal matrices whose $i$-th diagonal element is given by

$$
\alpha_{i}(\boldsymbol{x})=\alpha_{\lambda}\left(x_{i}, F_{i}(\boldsymbol{x})\right)-1, \quad \beta_{i}(\boldsymbol{x})=\beta_{\lambda}\left(x_{i}, F_{i}(\boldsymbol{x})\right)-1 .
$$

if $\left(x_{i}, F_{i}(\boldsymbol{x})\right) \neq(0,0)$, and by

$$
\alpha_{i}(\boldsymbol{x})=\xi_{i}-1, \quad \beta_{i}(\boldsymbol{x})=\chi_{i}-1,
$$

for every $\left(\xi_{i}, \chi_{i}\right) \in \mathbb{R}^{2}$ such that $\left\|\left(\xi_{i}, \chi_{i}\right)\right\| \leq 2-\frac{\lambda(4-\lambda)}{8}$, in the case $\left(x_{i}, F_{i}(\boldsymbol{x})\right)=(0,0)$.

Proof. It follows directly from the definition of the C-subdifferential and from [7, Proposition 2.5].

By the differentiabilty of $\Phi_{\lambda \mu}$ at $x_{k}$, the Jacobian matrix $\Phi_{\lambda \mu}^{\prime}\left(\boldsymbol{x}_{k}\right)$ is given by

$$
\Phi_{\lambda \mu}^{\prime}\left(\boldsymbol{x}_{k}\right)=\left(\begin{array}{c}
\nabla \varphi_{\lambda \mu}\left(x_{1}^{k}, F_{1}\left(\boldsymbol{x}_{k}\right)\right)^{T} \\
\vdots \\
\nabla \varphi_{\lambda \mu}\left(x_{n}^{k}, F_{n}\left(\boldsymbol{x}_{k}\right)\right)^{T}
\end{array}\right),
$$

where its $i$-th row $\nabla \varphi_{\lambda \mu}\left(x_{i}^{k}, F_{i}\left(\boldsymbol{x}_{k}\right)\right)^{T}$, denoted by $\left[\Phi_{\lambda \mu}^{\prime}\left(\boldsymbol{x}_{k}\right)\right]_{i}$, has the form

$$
\left[\Phi_{\lambda \mu}^{\prime}\left(\boldsymbol{x}_{k}\right)\right]_{i}=\left(\alpha_{\lambda \mu}\left(x_{i}^{k}, F_{i}\left(\boldsymbol{x}_{k}\right)\right)-1\right) \mathbf{e}_{i}^{T}+\left(\beta_{\lambda \mu}\left(x_{i}^{k}, F_{i}\left(\boldsymbol{x}_{k}\right)\right)-1\right) \nabla F_{i}\left(\boldsymbol{x}_{k}\right)^{T},
$$

with $\mathbf{e}_{1}, \ldots, \mathbf{e}_{n}$, the canonical vectors in $\mathbb{R}^{n}$, and where $\alpha_{\lambda \mu}$ and $\beta_{\lambda \mu}$ are the functions defined by (14), that is

$$
\begin{aligned}
& \alpha_{\lambda \mu}\left(x_{i}^{k}, F_{i}\left(\boldsymbol{x}_{k}\right)\right)=\frac{2\left(x_{i}^{k}-F_{i}\left(\boldsymbol{x}_{k}\right)\right)+\lambda F_{i}\left(\boldsymbol{x}_{k}\right)}{2 \sqrt{\left(x_{i}^{k}-F_{i}\left(\boldsymbol{x}_{k}\right)\right)^{2}+\lambda x_{i} F_{i}\left(\boldsymbol{x}_{k}\right)+(4-\lambda) \mu}}, \\
& \beta_{\lambda \mu}\left(x_{i}^{k}, F_{i}\left(\boldsymbol{x}_{k}\right)\right)=\frac{-2\left(x_{i}^{k}-F_{i}\left(\boldsymbol{x}_{k}\right)\right)+\lambda x_{i}^{k}}{2 \sqrt{\left(x_{i}^{k}-F_{i}\left(\boldsymbol{x}_{k}\right)\right)^{2}+\lambda x_{i} F_{i}\left(\boldsymbol{x}_{k}\right)+(4-\lambda) \mu}} .
\end{aligned}
$$

The next lemma guarantees that, as the parameter $\mu$ tends to zero, the distance between the matrix $\Phi_{\lambda \mu}^{\prime}(\boldsymbol{x})$ and the set $\partial_{C} \Phi_{\lambda}^{\prime}(\boldsymbol{x})$ also tends to zero. Therefore, it is reasonable to replace the generalized Newton iteration (5) by the iteration (22).

Lemma 3.3. Let $\boldsymbol{x} \in \mathbb{R}^{n}$ be arbitrary but fixed and $\mu>0$. Then we have

$$
\lim _{\mu \downarrow 0} \operatorname{dist}\left(\Phi_{\lambda \mu}^{\prime}(\boldsymbol{x}), \partial_{C} \Phi_{\lambda}(\boldsymbol{x})\right)=0 .
$$


Proof. Since

$$
\begin{aligned}
\lim _{\mu \downarrow 0} \operatorname{dist}\left(\Phi_{\lambda \mu}^{\prime}(\boldsymbol{x}), \partial_{C} \Phi_{\lambda}(\boldsymbol{x})\right) & =\lim _{\mu \downarrow 0} \inf _{H \in \partial_{C} \Phi_{\lambda}(\boldsymbol{x})}\left\|\Phi_{\lambda \mu}^{\prime}(\boldsymbol{x})-H\right\|_{F} \\
& =\inf _{H \in \partial_{C} \Phi_{\lambda}(\boldsymbol{x})} \lim _{\mu \downarrow 0}\left\|\Phi_{\lambda \mu}^{\prime}(\boldsymbol{x})-H\right\|_{F},
\end{aligned}
$$

in order to prove (23) it is enough to prove that is the limit of $\Phi_{\lambda \mu}^{\prime}(x)$ when $\mu \rightarrow 0$ is in $\partial_{C} \Phi_{\lambda}(x)$. To do this, let us define the index set $\Gamma(x)=$ $\left\{i: x_{i}=F_{i}(\boldsymbol{x})=0\right\}$. We have

$$
\begin{aligned}
\lim _{\mu \downarrow}[ & \left.\Phi_{\lambda \mu}^{\prime}(\boldsymbol{x})\right]_{i} \\
& = \begin{cases}\left(\alpha_{\lambda}\left(x_{i}, F_{i}(\boldsymbol{x})\right)-1\right) \boldsymbol{e}_{i}^{T}+\left(\beta_{\lambda}\left(x_{i}, F_{i}(\boldsymbol{x})\right)-1\right) \nabla F_{i}(\boldsymbol{x})^{T}, & i \notin \Gamma(\boldsymbol{x}) \\
-\boldsymbol{e}^{T}-\nabla F_{i}(\boldsymbol{x})^{T}, & i \in \Gamma(\boldsymbol{x})\end{cases} \\
=[H]_{i}, &
\end{aligned}
$$

then the matrix $H$ has the form described in Proposition 1 with $\left(\xi_{i}, \chi_{i}\right)=$ $(0,0)$, for $i \in \Gamma(x)$, therefore, $H \in \partial_{C} \Phi_{\lambda}(x)$. Thus, the infimun is zero and (23) is satisfied.

The following hypotheses allow to prove that the Algorithm 3.1 is well defined and converges to a solution of (8).

H1. The system (8) has a solution $\boldsymbol{x}_{*} \in \mathbb{R}^{n}$.

H2. The Jacobian matrix of $F$ is locally Lipschitz continuous.

H3. The matrices of $\partial_{C} \Phi_{\lambda}\left(\boldsymbol{x}_{*}\right)$ are nonsingular.

By the compactness of $\partial \Phi_{\lambda}\left(\boldsymbol{x}_{*}\right)$ (cf. [18]) and from H3, there is a constant $\beta$ such that

$$
\left\|H_{*}^{-1}\right\| \leq \beta
$$

for all $H_{*} \in \partial_{C} \Phi_{\lambda}\left(\boldsymbol{x}_{*}\right)$.

Next, we present a technical lemma that will be useful in the proof of Lemma 3.5.

Lemma 3.4. Assume $\mathrm{H} 1$ and $\mathrm{H} 3$, and $r \in(0,1)$. There exists a positive constant $\epsilon$ such that, if $\left\|\boldsymbol{x}-\boldsymbol{x}_{*}\right\|_{\infty}<\epsilon$ then the function $\mathscr{Q}$ defined by

$$
\mathscr{Q}(\boldsymbol{x})=\boldsymbol{x}-\Phi_{\lambda \mu}^{\prime}(\boldsymbol{x})^{-1} \Phi_{\lambda}(\boldsymbol{x})
$$

is well defined, and

$$
\left\|\mathscr{Q}(\boldsymbol{x})-\boldsymbol{x}_{*}\right\|_{\infty} \leq r\left\|\boldsymbol{x}-\boldsymbol{x}_{*}\right\|_{\infty} .
$$


Moreover, if the Jacobian matrix of $F$ satisfies Assumption $\mathrm{H} 2$ then

$$
\left\|\mathscr{Q}(\boldsymbol{x})-\boldsymbol{x}_{*}\right\|_{\infty} \leq c\left\|\boldsymbol{x}-\boldsymbol{x}_{*}\right\|_{\infty}^{2} .
$$

Proof. In order to prove that $\Phi_{\lambda \mu}^{\prime}(\boldsymbol{x})$ is nonsingular, we use the Banach's Lemma [14]. To do this, we find a bound for $\left\|\Phi_{\lambda \mu}^{\prime}(\boldsymbol{x})-H_{*}\right\|$, where $H_{*} \in$ $\partial_{C} \Phi_{\lambda}\left(\boldsymbol{x}_{*}\right)$. Using the definition of C-subdifferential, we have that the matrix $H_{*}$ has the form

$$
H_{*}=\left(\begin{array}{c}
{\left[H_{*}\right]_{1}} \\
\vdots \\
{\left[H_{*}\right]_{n}}
\end{array}\right)=\lim _{k \rightarrow \infty} \Phi_{\lambda}^{\prime}\left(\boldsymbol{y}^{k}\right)=\left(\begin{array}{c}
\lim _{k \rightarrow \infty} \nabla \varphi_{\lambda}\left(y_{1}^{k}, F_{1}\left(\boldsymbol{y}^{k}\right)\right)^{T} \\
\vdots \\
\lim _{k \rightarrow \infty} \nabla \varphi_{\lambda}\left(y_{n}^{k}, F_{n}\left(\boldsymbol{y}^{k}\right)\right)^{T}
\end{array}\right),
$$

where the sequence $\left\{\boldsymbol{y}^{k}\right\}$ converges to $\boldsymbol{x}_{*}$ and satisfies that $\Phi_{\lambda}^{\prime}\left(\boldsymbol{y}^{k}\right)$ exists. We bound $\left\|\Phi_{\lambda \mu}^{\prime}(\boldsymbol{x})-H_{*}\right\|$ using the definition of infinite matrix norm. Thus, for some $j \in\{1,2, \ldots, n\}$, we have

$$
\begin{aligned}
\left\|\Phi_{\lambda \mu}^{\prime}(\boldsymbol{x})-H_{*}\right\|_{\infty} & =\left\|\left[\Phi_{\lambda \mu}^{\prime}\left(\boldsymbol{x}_{*}\right)\right]_{j}-\left[H_{*}\right]_{j}\right\|_{1} \\
& \leq n\left\|\nabla \varphi_{\lambda \mu}\left(x_{j}, F_{j}(\boldsymbol{x})\right)^{T}-\lim _{k \rightarrow \infty} \nabla \varphi_{\lambda}\left(y_{j}^{k}, F_{j}\left(\boldsymbol{y}^{k}\right)\right)^{T}\right\|_{\infty} \\
& \leq n \lim _{k \rightarrow \infty}\left\|\nabla \varphi_{\lambda \mu}\left(x_{j}, F_{j}(\boldsymbol{x})\right)^{T}-\nabla \varphi_{\lambda}\left(y_{j}^{k}, F_{j}\left(\boldsymbol{y}^{k}\right)\right)^{T}\right\|_{\infty} .
\end{aligned}
$$

By Lemma 2.7, we have

$$
\begin{aligned}
\left\|\Phi_{\lambda \mu}^{\prime}(\boldsymbol{x})-H_{*}\right\|_{\infty} & \leq n \tau \lim _{k \rightarrow \infty}\left\|\left(\begin{array}{c}
x_{j}-y_{j}^{k} \\
F_{j}(\boldsymbol{x})-F_{j}\left(\boldsymbol{y}^{k}\right)
\end{array}\right)\right\| \\
& \leq \sqrt{2} n \tau\left\|\left(\begin{array}{c}
x_{j}-x_{j}^{*} \\
F_{j}(\boldsymbol{x})-F_{j}\left(\boldsymbol{x}_{*}\right)
\end{array}\right)\right\|_{\infty} \\
& \leq \sqrt{2} n \tau \max \left\{\left|x_{j}-x_{j}^{*}\right|,\left|F_{j}(\boldsymbol{x})-F_{j}\left(\boldsymbol{x}_{*}\right)\right|\right\} .
\end{aligned}
$$

By de continuity of $F$, for all $\hat{\epsilon}$, there exists $\hat{\delta}>0$ such that, if we have $\left\|\boldsymbol{x}-\boldsymbol{x}^{*}\right\|_{\infty}<\hat{\delta}$ then $\left|F_{j}(\boldsymbol{x})-F_{j}\left(\boldsymbol{x}^{*}\right)\right|<\hat{\epsilon}$. Let $\epsilon^{\prime}=\min \{\hat{\epsilon}, \hat{\delta}\}$. We consider the two possibilities for the maximum in (28).

1. If $\max \left\{\left|x_{j}-x_{j}^{*}\right|,\left|F_{j}(\boldsymbol{x})-F_{j}\left(\boldsymbol{x}_{*}\right)\right|\right\}=\left|x_{j}-x_{j}^{*}\right| \leq\left\|\boldsymbol{x}-\boldsymbol{x}_{*}\right\|_{\infty} \leq \epsilon^{\prime}<\hat{\epsilon}$.

2. If $\max \left\{\left|x_{j}-x_{j}^{*}\right|,\left|F_{j}(\boldsymbol{x})-F_{j}\left(\boldsymbol{x}_{*}\right)\right|\right\}=\left|F_{j}\left(\boldsymbol{x}^{k}\right)-F_{j}\left(\boldsymbol{x}_{*}\right)\right|<\hat{\epsilon}$. 
Thus, from (28), for any $\hat{\epsilon}$

$$
\left\|\Phi_{\lambda \mu}^{\prime}(x)-H_{*}\right\|_{\infty}<\sqrt{2} n \tau \hat{\epsilon} .
$$

Let $\hat{\epsilon}<\frac{1}{2 \sqrt{2} \rho n \beta}$, then $\left\|\Phi_{\lambda \mu}^{\prime}(\boldsymbol{x})-H_{*}\right\|_{\infty} \leq \tau n \hat{\epsilon}<\frac{1}{2 \beta}$. Now,

$$
\left\|H_{*}^{-1} \Phi_{\lambda \mu}^{\prime}(\boldsymbol{x})-I\right\|_{\infty} \leq\left\|H_{*}^{-1}\right\|_{\infty}\left\|\Phi_{\lambda \mu}^{\prime}(\boldsymbol{x})-H_{*}\right\|_{\infty} \leq \beta \frac{1}{2 \beta}=\frac{1}{2},
$$

thus $\left\|H_{*}^{-1} \Phi_{\lambda \mu}^{\prime}(\boldsymbol{x})-I\right\|_{\infty}<1$; therefore, there exists $\Phi_{\lambda \mu}^{\prime}(\boldsymbol{x})^{-1}$ (Banach's Lemma [14]) and the function $\mathscr{Q}$ is well defined. In addition,

$$
\left\|\Phi_{\lambda \mu}^{\prime}(\boldsymbol{x})^{-1}\right\|_{\infty} \leq \frac{\left\|H_{*}^{-1}\right\|_{\infty}}{1-\left\|H_{*}^{-1} \Phi_{\lambda \mu}^{\prime}(\boldsymbol{x})-I_{n}\right\|_{\infty}} \leq \frac{\beta}{1-\frac{1}{2}}=2 \beta .
$$

In the second part of the proof, we prove (26). For this, subtracting $\boldsymbol{x}_{*}$ in (25), using $\|\cdot\|_{\infty}$, and performing some algebraic manipulations, we have

$$
\begin{aligned}
& \| \\
& \quad=\left\|\Phi_{\lambda \mu}^{\prime}(\boldsymbol{x})^{-1}\left[\Phi_{\lambda \mu}^{\prime}(\boldsymbol{x})\left(\boldsymbol{x}-\boldsymbol{x}_{*}\right)-\Phi_{\lambda}(\boldsymbol{x})+\Phi_{\lambda}\left(\boldsymbol{x}_{*}\right)\right]\right\|_{\infty} \\
& \leq\left\|\Phi_{\lambda \mu}^{\prime}(\boldsymbol{x})^{-1}\right\|_{\infty}\left\|\Phi_{\lambda \mu}^{\prime}(\boldsymbol{x})\left(\boldsymbol{x}-\boldsymbol{x}_{*}\right)-\Phi_{\lambda}(\boldsymbol{x})+\Phi_{\lambda}\left(\boldsymbol{x}_{*}\right)\right\|_{\infty} \\
& \leq 2 \beta\left\|\Phi_{\lambda \mu}^{\prime}(\boldsymbol{x})\left(\boldsymbol{x}-\boldsymbol{x}_{*}\right)-H\left(\boldsymbol{x}-\boldsymbol{x}_{*}\right)+H\left(\boldsymbol{x}-\boldsymbol{x}_{*}\right)-\Phi_{\lambda}(\boldsymbol{x})+\Phi_{\lambda}\left(\boldsymbol{x}_{*}\right)\right\|_{\infty} \\
& \leq 2 \beta\left[\left\|\Phi_{\lambda \mu}^{\prime}(\boldsymbol{x})-H\right\|\left\|\boldsymbol{x}-\boldsymbol{x}_{*}\right\|_{\infty}+\left\|H\left(\boldsymbol{x}-\boldsymbol{x}_{*}\right)-\Phi_{\lambda}(\boldsymbol{x})+\Phi_{\lambda}\left(\boldsymbol{x}_{*}\right)\right\|_{\infty}\right]
\end{aligned}
$$

By Lemma 3.3, for all $\delta>0$, there exists $\epsilon_{1}>0$ such that, if $\left\|\boldsymbol{x}-\boldsymbol{x}_{*}\right\|_{\infty}<\epsilon_{1}$,

$$
\left\|\Phi_{\lambda \mu}^{\prime}(\boldsymbol{x})-H\right\|<\delta .
$$

thus, for $\delta<\frac{r}{4 \beta}$, there exists $\epsilon_{r}>0$ such that, if $\left\|\boldsymbol{x}-\boldsymbol{x}_{*}\right\|_{\infty}<\epsilon_{r}$,

$$
\left\|\Phi_{\lambda \mu}^{\prime}(\boldsymbol{x})-H\right\|<\frac{r}{4 \beta}
$$

Moreover, by Theorem 2.3 in [7], for all $\rho>0$, there exists $\epsilon_{2}>0$, such that, if $\left\|\boldsymbol{x}-\boldsymbol{x}_{*}\right\|_{\infty}<\epsilon_{1}$ then $\left\|H\left(\boldsymbol{x}-\boldsymbol{x}_{*}\right)-\Phi_{\lambda}(\boldsymbol{x})+\Phi_{\lambda}\left(\boldsymbol{x}_{*}\right)\right\|_{\infty}<\rho\left\|\boldsymbol{x}-\boldsymbol{x}_{*}\right\|_{\infty}$. In particular, for $\rho<\frac{r}{4 \beta}$, there exists $\bar{\epsilon}_{r}>0$ such that, if $\left\|\boldsymbol{x}-\boldsymbol{x}_{*}\right\|_{\infty}<\bar{\epsilon}_{r}$ then

$$
\left\|H\left(\boldsymbol{x}-\boldsymbol{x}_{*}\right)-\Phi_{\lambda}(\boldsymbol{x})+\Phi_{\lambda}\left(\boldsymbol{x}_{*}\right)\right\|_{\infty}<\frac{r}{4 \beta}\left\|\boldsymbol{x}-\boldsymbol{x}_{*}\right\|_{\infty} .
$$


Thus, for $\epsilon=\min \left\{\epsilon^{\prime}, \epsilon_{r}, \bar{\epsilon}_{r}\right\}$, we have (29), (30), and (31), therefore

$$
\left\|\mathscr{Q}(\boldsymbol{x})-\boldsymbol{x}_{*}\right\|_{\infty} \leq r\left\|\boldsymbol{x}-\boldsymbol{x}_{*}\right\|_{\infty}
$$

and we have proved (26).

The third part of the proof consists in obtaining (27) under the hypothesis H2. By [7, Theorem 2.3], there exists a positive constant $\gamma$ such that

$$
\left\|H\left(\boldsymbol{x}-\boldsymbol{x}_{*}\right)-\Phi_{\lambda}(\boldsymbol{x})+\Phi_{\lambda}\left(\boldsymbol{x}_{*}\right)\right\|_{\infty} \leq \gamma\left\|\boldsymbol{x}-\boldsymbol{x}_{*}\right\|_{\infty}^{2} .
$$

On the other hand, adding and subtracting $H_{*}$ in $\left\|\Phi_{\lambda \mu}^{\prime}(\boldsymbol{x})-H\right\|_{\infty}$ and using the triangle inequality, we have

$$
\left\|\Phi_{\lambda \mu}^{\prime}(\boldsymbol{x})-H\right\|_{\infty} \leq\left\|\Phi_{\lambda \mu}^{\prime}(\boldsymbol{x})-H_{*}\right\|_{\infty}+\left\|H_{*}-H\right\|_{\infty} .
$$

By (28), a bound for the first term of (33) is $\sqrt{2} n \tau M$, where

$$
M=\max \left\{\left|x_{j}-x_{j}^{*}\right|,\left|F_{j}(\boldsymbol{x})-F_{j}\left(\boldsymbol{x}_{*}\right)\right|\right\},
$$

and by [23, Lemma 4.2] the second term is bounded by $\sqrt{2} n \eta M$, where $\eta$ is the Lipschitz constant of $\nabla \varphi_{\lambda}$. Then,

$$
\left\|\Phi_{\lambda \mu}^{\prime}(\boldsymbol{x})-H\right\|_{\infty} \leq \sqrt{2} n(\tau+\eta) M
$$

Moreover,

1. If $M=\left|x_{j}-x_{j}^{*}\right|$ then $M \leq\left\|\boldsymbol{x}-\boldsymbol{x}_{*}\right\|_{\infty}$.

2. If $M=\left|F_{j}\left(\boldsymbol{x}_{k}\right)-F_{j}\left(\boldsymbol{x}_{*}\right)\right|$ then $M \leq\left\|F\left(\boldsymbol{x}_{k}\right)-F\left(\boldsymbol{x}_{*}\right)\right\|_{\infty} \leq \zeta\left\|\boldsymbol{x}_{k}-\boldsymbol{x}_{*}\right\|_{\infty}$, where the last inequality is given by [14, Lemma 4.1.16], because $F$ is continuously differentiable and its Jacobian matrix is Lipschitz continuous in a neighborhood of $\boldsymbol{x}_{*}$.

Let $\bar{\zeta}=\max \{1, \zeta\}$. From $(34)$ and the previous cases about $M$,

$$
\left\|\Phi_{\lambda \mu}^{\prime}(\boldsymbol{x})-H\right\|_{\infty} \leq \sqrt{2} n(\tau+\eta) \bar{\zeta}\left\|\boldsymbol{x}_{k}-\boldsymbol{x}_{*}\right\|_{\infty}=\theta\left\|\boldsymbol{x}_{k}-\boldsymbol{x}_{*}\right\|_{\infty}
$$

with $\theta=\sqrt{2} n(\tau+\eta) \bar{\zeta}$. Thus, from (29), (32), and (35),

$\left\|\mathscr{Q}(\boldsymbol{x})-\boldsymbol{x}_{*}\right\|_{\infty} \leq 2 \beta\left[\theta\left\|\boldsymbol{x}-\boldsymbol{x}_{*}\right\|_{\infty}^{2}+\gamma\left\|\boldsymbol{x}-\boldsymbol{x}_{*}\right\|_{\infty}^{2}\right] \leq 2 \beta(\theta+\gamma)\left\|\boldsymbol{x}-\boldsymbol{x}_{*}\right\|_{\infty}^{2}$.

Therefore,

$$
\left\|\mathscr{Q}(\boldsymbol{x})-\boldsymbol{x}_{*}\right\|_{\infty} \leq c\left\|\boldsymbol{x}-\boldsymbol{x}_{*}\right\|_{\infty}^{2},
$$

with $c=2 \beta(\theta+\gamma)$. 
The following lemma guarantees that the proposed algorithm is well defined, it converges linearly and it gives a sufficient condition for $q$-quadratic convergence.

Lemma 3.5. There exists a positive $\epsilon_{0}$ such that, if $\left\|x_{0}-x_{*}\right\|_{\infty}<\epsilon_{0}$ then

$$
\boldsymbol{x}_{k+1}=\boldsymbol{x}_{k}-\Phi_{\lambda \mu}^{\prime}\left(\boldsymbol{x}_{k}\right)^{-1} \Phi_{\lambda}\left(\boldsymbol{x}_{k}\right)
$$

generates a well defined sequence $\left\{\boldsymbol{x}_{k}\right\}$ which converges to $\boldsymbol{x}_{*}$, and given $r \in(0,1)$, satisfies

$$
\left\|\boldsymbol{x}_{k+1}-\boldsymbol{x}_{*}\right\|_{\infty} \leq r\left\|\boldsymbol{x}_{k}-\boldsymbol{x}_{*}\right\|_{\infty} .
$$

Moreover, if the Jacobian matrix of $F$ satisfies the assumption $\mathrm{H} 2$ then

$$
\left\|\boldsymbol{x}_{k+1}-\boldsymbol{x}_{*}\right\|_{\infty} \leq c\left\|\boldsymbol{x}_{k}-\boldsymbol{x}_{*}\right\|_{\infty}^{2}
$$

where $c$ is the constant of Lemma 3.4 .

Proof. Let $\mathscr{Q}$ be defined in (25). Thus, for $k=0,1, \ldots$

$$
\boldsymbol{x}_{k+1}=\mathscr{Q}\left(\boldsymbol{x}_{k}\right)=\boldsymbol{x}_{k}-\Phi_{\lambda \mu}^{\prime}\left(\boldsymbol{x}_{k}\right)^{-1} \Phi_{\lambda}\left(\boldsymbol{x}_{k}\right) .
$$

Let $r \in(0,1)$ and $\epsilon_{0} \in(0, \epsilon)$, where $\epsilon$ is the constant of Lemma 3.3. The proof is by induction on $k$.

- For $k=0$, if $\left\|\boldsymbol{x}_{0}-\boldsymbol{x}_{*}\right\|_{\infty} \leq \epsilon_{0}<\epsilon$, by Lemma 3.3, $x_{1}=\mathscr{Q}\left(x_{0}\right)$ is well defined and verifies

$$
\left\|\boldsymbol{x}_{1}-\boldsymbol{x}_{*}\right\|_{\infty} \leq r\left\|\boldsymbol{x}_{0}-\boldsymbol{x}_{*}\right\|_{\infty} .
$$

Moreover, if the Jacobian matrix of $F$ is Lipschitz continuous in a neighborhood of $\boldsymbol{x}_{*}$,

$$
\left\|\boldsymbol{x}_{1}-\boldsymbol{x}_{*}\right\|_{\infty} \leq c\left\|\boldsymbol{x}_{0}-\boldsymbol{x}_{*}\right\|_{\infty}^{2} .
$$

- Induction hypotheses. For all $0<k \leq m-1$, we have $\left\|\boldsymbol{x}_{k}-\boldsymbol{x}_{*}\right\|_{\infty}<\epsilon_{0}$. Then, by Lemma 3.3, $\boldsymbol{x}_{m}=\mathscr{Q}\left(\boldsymbol{x}_{m-1}\right)$ is well defined and

$$
\left\|\boldsymbol{x}_{m}-\boldsymbol{x}^{*}\right\|_{\infty}=\left\|\mathscr{Q}\left(\boldsymbol{x}_{m-1}\right)-\boldsymbol{x}_{*}\right\|_{\infty} \leq r\left\|\boldsymbol{x}_{m-1}-\boldsymbol{x}^{*}\right\|_{\infty} .
$$

Moreover, $\left\|\boldsymbol{x}_{m}-\boldsymbol{x}_{*}\right\|_{\infty} \leq c\left\|\boldsymbol{x}_{m-1}-\boldsymbol{x}_{*}\right\|_{\infty}^{2}$, if the Jacobian matrix of $F$ is Lipschitz continuous at $\boldsymbol{x}_{*}$.

From (39), we have,

$$
\left\|\boldsymbol{x}_{m}-\boldsymbol{x}_{*}\right\|_{\infty} \leq r\left\|\boldsymbol{x}_{m-1}-\boldsymbol{x}_{*}\right\|_{\infty} \leq r^{m}\left\|\boldsymbol{x}_{0}-\boldsymbol{x}_{*}\right\|_{\infty} \leq r^{m} \epsilon_{0}<\epsilon,
$$


thus, Lemma 3.3 guarantees that $\boldsymbol{x}_{m+1}$ is well defined and satisfies

$$
\left\|\boldsymbol{x}_{m+1}-\boldsymbol{x}_{*}\right\|_{\infty} \leq r\left\|\boldsymbol{x}_{m}-\boldsymbol{x}_{*}\right\|_{\infty},
$$

and, if H2 is verified then $\boldsymbol{x}_{m+1}$ satisfies

$$
\left\|\boldsymbol{x}_{m+1}-\boldsymbol{x}_{*}\right\|_{\infty} \leq c\left\|\boldsymbol{x}_{m}-\boldsymbol{x}_{*}\right\|_{\infty}^{2} .
$$

Therefore, we conclude that (37) and (38) are true for all $k=0,1, \ldots$

\section{Numerical experiments}

In this section, we analyze numerically the Jacobian smoothing algorithm introduced in the previous section (Algorithm 1). To do this, we compared our algorithm with the one proposed in [17] (which is a particular case of our algorithm when $\lambda=2$ ), which we call Algorithm 2, and with a Neroton method that uses matrices in the C-subdifferential of $\Phi_{\lambda}$ at $x_{k}$.

For the variation of parameter $\mu$, we consider the sequences $\left\{\mu_{0} 2^{-k}\right\}$ and $\left\{\mu_{0} 100^{-k}\right\}$, with $\mu_{0}=\frac{\alpha}{2 \varkappa}, x=\sqrt{2 n}, \alpha \in(0,1)$ given in [17]. Moreover, in both, the proposed algorithm and in the Newton method, the parameter $\lambda$ varies from $\lambda=10^{-1}$ to $\lambda=3.9$ with increments of $10^{-1}$. It is important to mention that in the Algorithm 2 proposed in [17], $\lambda=2$ and $\mu$ depend on the function $F$.

We use five test problems, four of them were chosen from [6]. The fifth function is [24, Example A]. We describe each of these functions below, as well as the initial point used $\left(x_{0}\right)$ and the solutions found $\left(x_{*}\right)$.

1. Billups problem [6]. Let $F: \mathbb{R} \rightarrow \mathbb{R}$ be defined by

$$
\begin{aligned}
& F(x)=(x-1)^{2}-1.1, \\
x_{0}=0 & \text { and } x_{*}=2.0488 .
\end{aligned}
$$

2. Mathiesen-modified problem [6]. Let $F: \mathbb{R}^{4} \rightarrow \mathbb{R}^{4}$ be defined by

$$
\begin{gathered}
F(\boldsymbol{x})=\left(\begin{array}{c}
-x_{2}+x_{3}+x_{4} \\
x_{1}-\frac{4.5 x_{3}+2.7 x_{4}}{x_{2}+1} \\
5-x_{1}-\frac{0.5 x_{3}+0.3 x_{4}}{x_{3}+1} \\
3-x_{1}
\end{array}\right), \\
x_{0}=\left(\begin{array}{llll}
1 & 1 & 1 & 1
\end{array}\right)^{T} \text { and } x_{*}=\left(\begin{array}{llll}
a & 0 & 0
\end{array}\right)^{T} \text {, with } a \in[0,3] .
\end{gathered}
$$


3. Kojima-Shindo problem [6]. Let $F: \mathbb{R}^{4} \rightarrow \mathbb{R}^{4}$ be defined by

$$
\begin{gathered}
F(\boldsymbol{x})=\left(\begin{array}{c}
3 x_{1}^{2}+2 x_{1} x_{2}+2 x_{2}^{2}+x_{3}+3 x_{4}-6 \\
2 x_{1}^{2}+x_{2}^{2}+x_{1}+10 x_{3}+2 x_{4}-2 \\
3 x_{1}^{2}+x_{1} x_{2}+2 x_{2}^{2}+2 x_{3}+9 x_{4}-9 \\
x_{1}^{2}+3 x_{2}^{2}+2 x_{3}+3 x_{4}-3
\end{array}\right), \\
x_{0}=\left(\begin{array}{llll}
1 & 1 & 1 & 1
\end{array}\right)^{T} \text { and } \boldsymbol{x}_{*}=\left(\begin{array}{llll}
\sqrt{6} / 2 & 0 & 0 & 1 / 2
\end{array}\right)^{T} .
\end{gathered}
$$

4. Kojima-Josephy problem [6]. $F: \mathbb{R}^{4} \rightarrow \mathbb{R}^{4}$ be defined by

$$
\begin{gathered}
F(\boldsymbol{x})=\left(\begin{array}{c}
3 x_{1}^{2}+2 x_{1} x_{2}+2 x_{2}^{2}+x_{3}+3 x_{4}-6 \\
2 x_{1}^{2}+x_{2}^{2}+x_{1}+3 x_{3}+2 x_{4}-2 \\
3 x_{1}^{2}+x_{1} x_{2}+2 x_{2}^{2}+2 x_{3}+3 x_{4}-9 \\
x_{1}^{2}+3 x_{2}^{2}+2 x_{3}+3 x_{4}-3
\end{array}\right), \\
x_{0}=\left(\begin{array}{llll}
1 & 1 & 1 & 1
\end{array}\right)^{T} \text { and } x_{*}=\left(\begin{array}{llll}
\sqrt{6} / 2 & 0 & 0 & 1 / 2
\end{array}\right)^{T} .
\end{gathered}
$$

5. Example $A$ in [24]. $F: \mathbb{R}^{5} \rightarrow \mathbb{R}^{5}$ be defined by

$$
\begin{gathered}
F(\boldsymbol{x})=\left(\begin{array}{c}
x_{1}+x_{2} x_{3} x_{4} x_{5} / 50 \\
x_{2}+x_{1} x_{3} x_{4} x_{5} / 50-3 \\
x_{3}+x_{1} x_{2} x_{4} x_{5} / 50-1 \\
x_{4}+x_{1} x_{2} x_{3} x_{5} / 50-0.5 \\
x_{5}+x_{1} x_{2} x_{3} x_{4} / 50
\end{array}\right), \\
x_{0}=\left(\begin{array}{lllll}
1 & -1 & 2-2 & 5
\end{array}\right)^{T} \text { and } x_{*}=\left(\begin{array}{lllll}
0 & 3 & 1 & 0
\end{array}\right)^{T} .
\end{gathered}
$$

To write the codes of the algorithms and the test functions, we used the software MATLAB ${ }^{\circledR}$ and we performed the numerical tests on a computer with a processor Intel(R) Core(TM) i5-4200M CPU@2.50 GHZ. We used as convergence criterion $\left\|\Phi_{\lambda}\left(x_{k}\right)\right\|<10^{-6}$ or $\left\|\Phi_{\lambda \mu}\left(x_{k}\right)\right\|<10^{-6}$, according to the case. We declared divergence if the number of iterations exceeded 500.

For each problem and with each algorithm, we compared the number of iterations and the time (in seconds) used by the algorithm to obtain convergence for each value of $\lambda$. The results obtained are presented below in ten graphics.

Fig. 1 shows that the Newton method converges only for $\lambda \geq 3.3$, with more iterations than the proposed algorithm, which converges for $\lambda \geq 2$, and the number of iterations is the same for the two sequences $\left\{\mu_{k}\right\}$ used, therefore its graphics match (Fig. 1A). On the other hand, Algorithm 2 diverges. In addition, Algorithm 1 is the most efficient. 
A

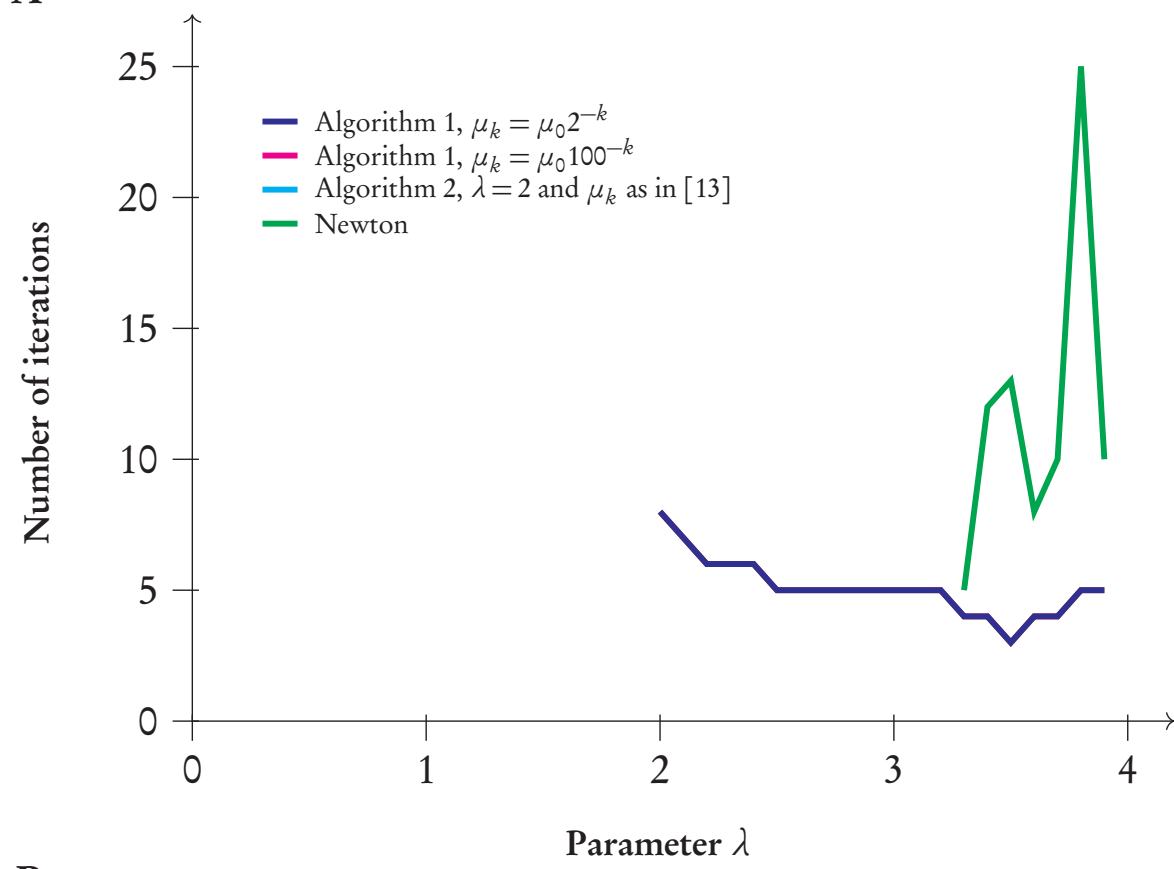

B

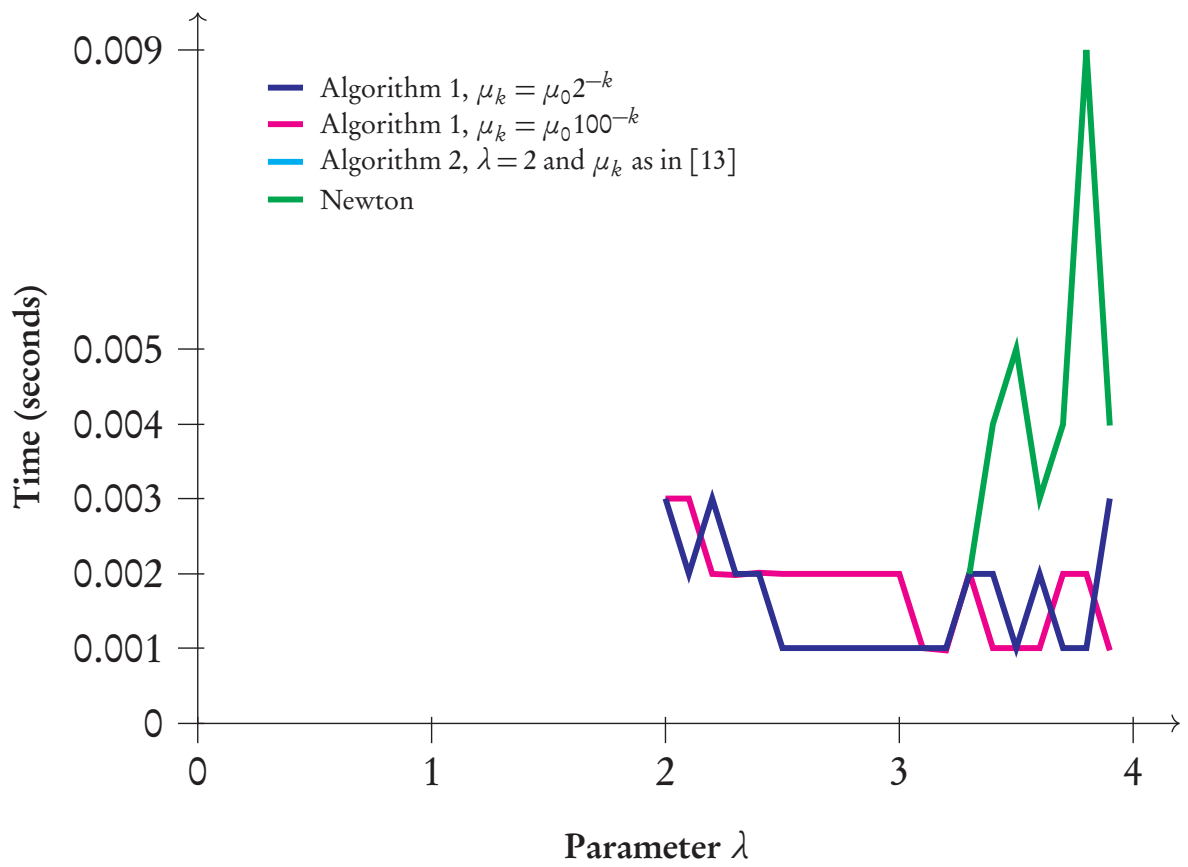

Figure 1. For the Billups function, the Algorithm 2 has a better performance in both number of iterations (A) and computational time (B). For $\lambda \in[0,2)$ there is no graph because Algorithms 1 and 2 diverge.

Fig. 2 shows that convergence was obtained for all the algorithms and all the values of $\lambda$. The number of iterations for the Nerwton method is slightly smaller than the others ones (Fig. 2A); the proposed smoothing has a fairly competitive behavior. In terms of computational time, the proposed algorithm lies between the generalized Newton method and the Algorithm 2 (Fig. 2B). 


\section{A}

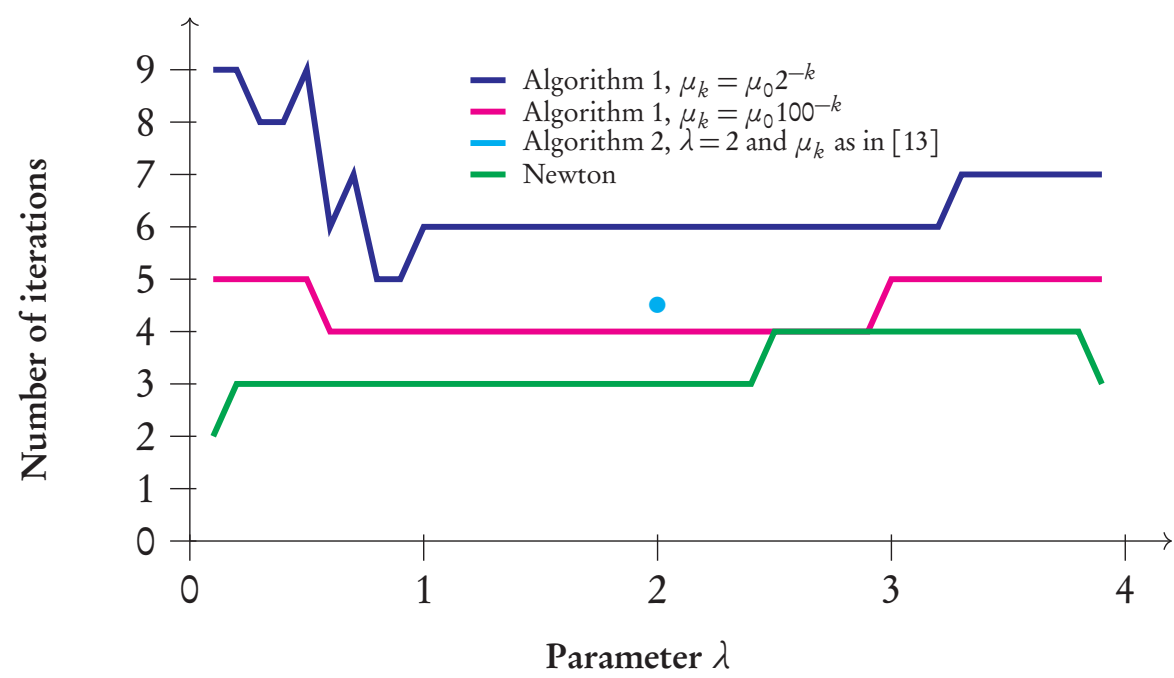

B

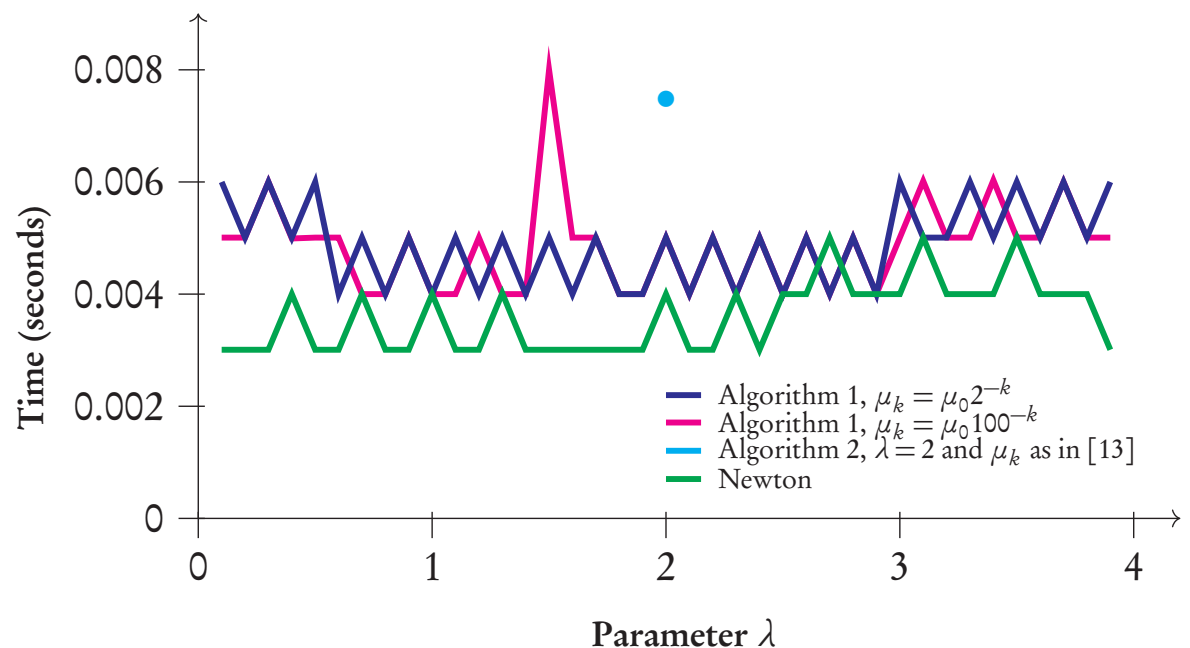

Figure 2. For the Mathiesen function, the algorithms are competitive in number of iterations (A) and computational time (B).

In Fig.3, we observe that the generalized Newton method converges for $\lambda \geq$ 1.8 , and the proposed algorithm converges for $\lambda \geq 1.5$; in terms of number of iterations and convergence time, these two methods have a similar behavior for $1.5 \leq \lambda \leq 3.1$. For $\lambda>3.1$ the proposed algorithm is the best.

Fig. 4 shows that the generalized Newton method and the proposed algorithm converge for $\lambda \geq 2$; the number of iterations is the same for the two sequences $\left\{\mu_{k}\right\}$ used and for the generalized Newton method; In addition, the Algorithm 2 converges using more iterations and spending more computational time than the other methods. 


\section{A}

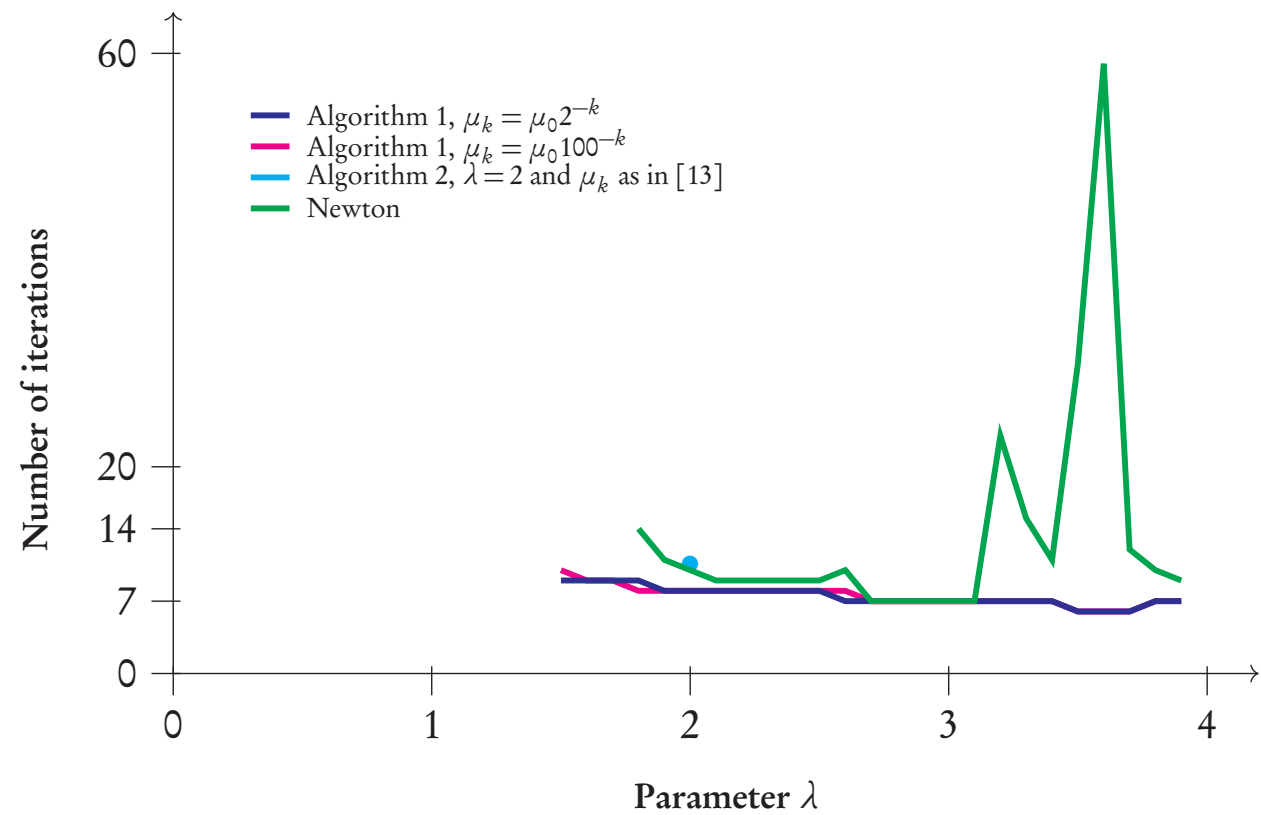

B

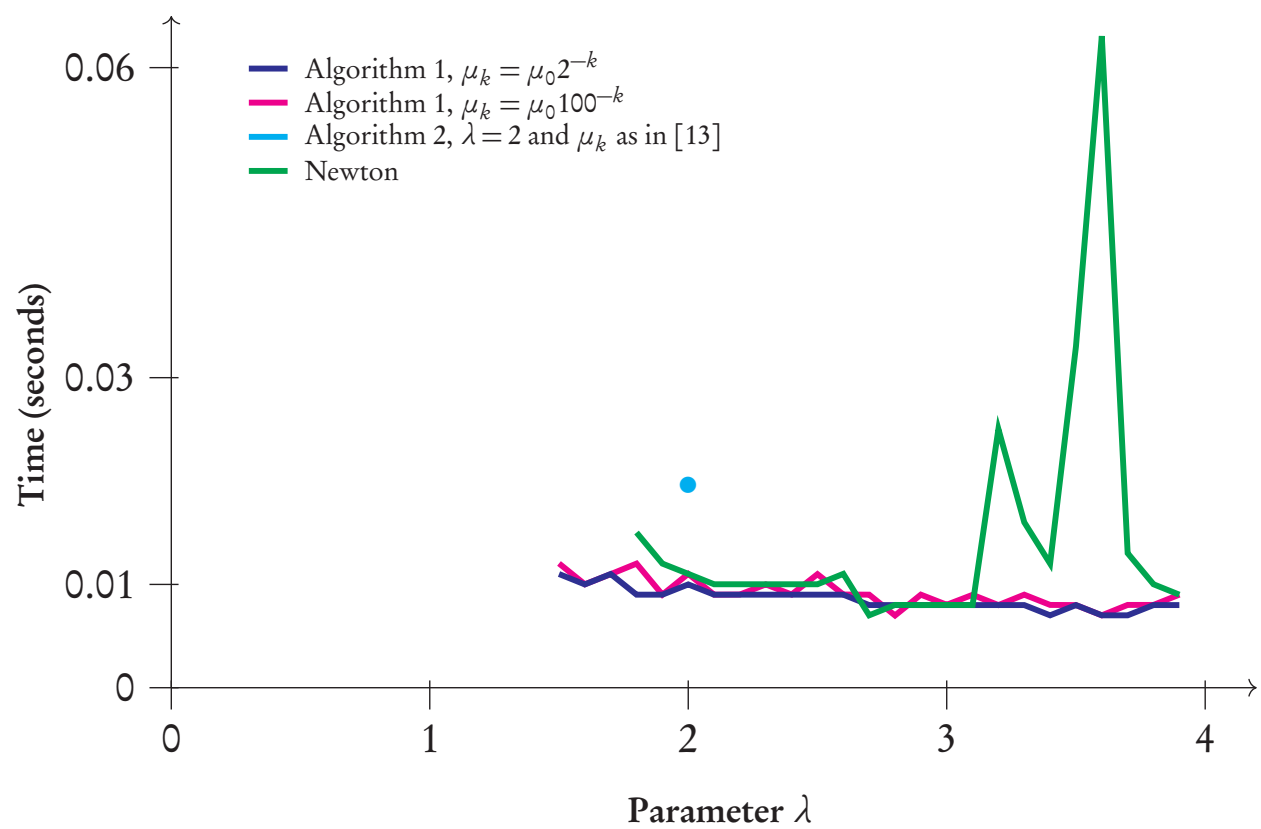

Figure 3. For the Kojima-Shindo function, the algorithm proposed is better than the other ones from $\lambda>3.1$ in both number of iterations (A) and computational time (B). For $\lambda \in[0,1.8)$ there are no graphs because Algorithms 1 and 2 diverge.

Finally, in Fig.5, we observe convergence of all the considered methods, for all values of $\lambda$. Again, it is shown that the proposed algorithm, Algorithm 1, is quite competitive with respect to the Newton method. On the other hand, in our proposal, the selection of the sequence $\mu_{k}$ has an important role in the convergence rate. 
A

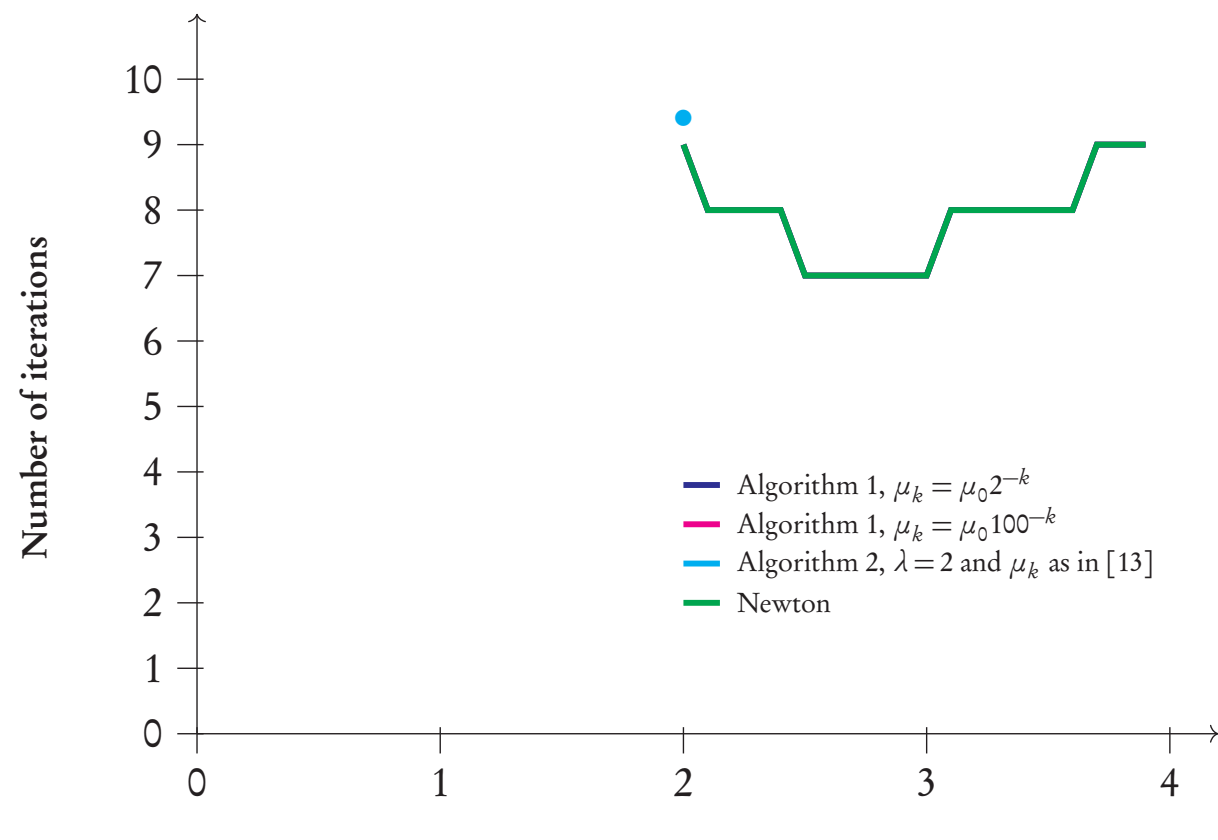

Parameter $\lambda$

B

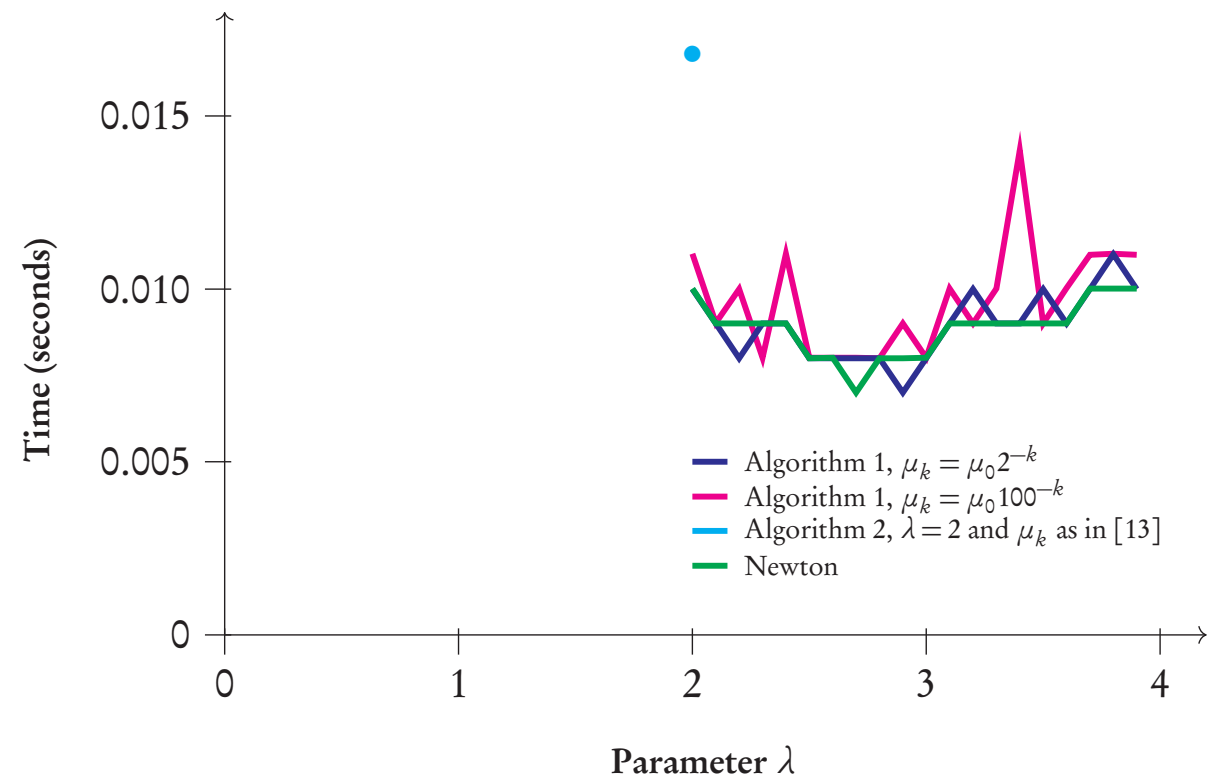

Figure 4. For Kojima-Josephy function, Algorithm 1 (with two sequences used) and the Nerton method have the same number of iterations, for this reason their graphics coincide (A). The Algorithm 2 is slower computationally (B). In addition, for $\lambda \in[0,2)$ there is no graph because Algorithms 1 and 2 diverge. 
A

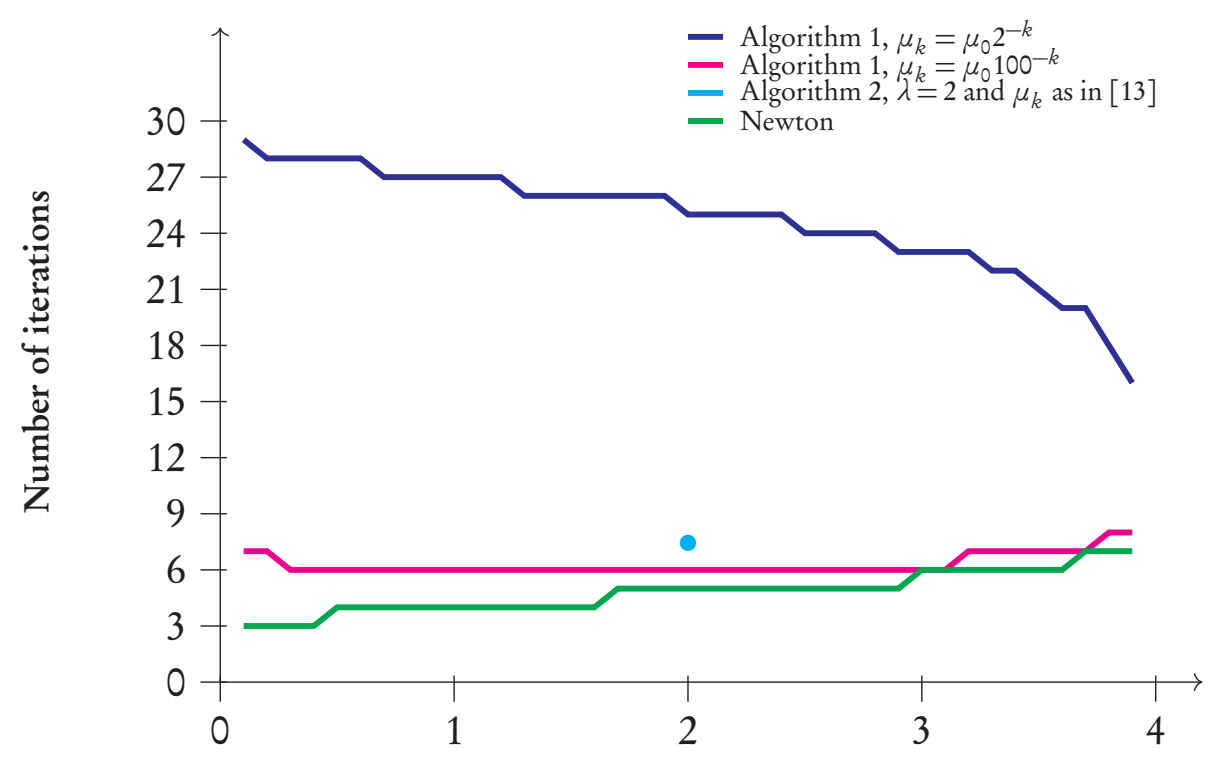

B

Parameter $\lambda$

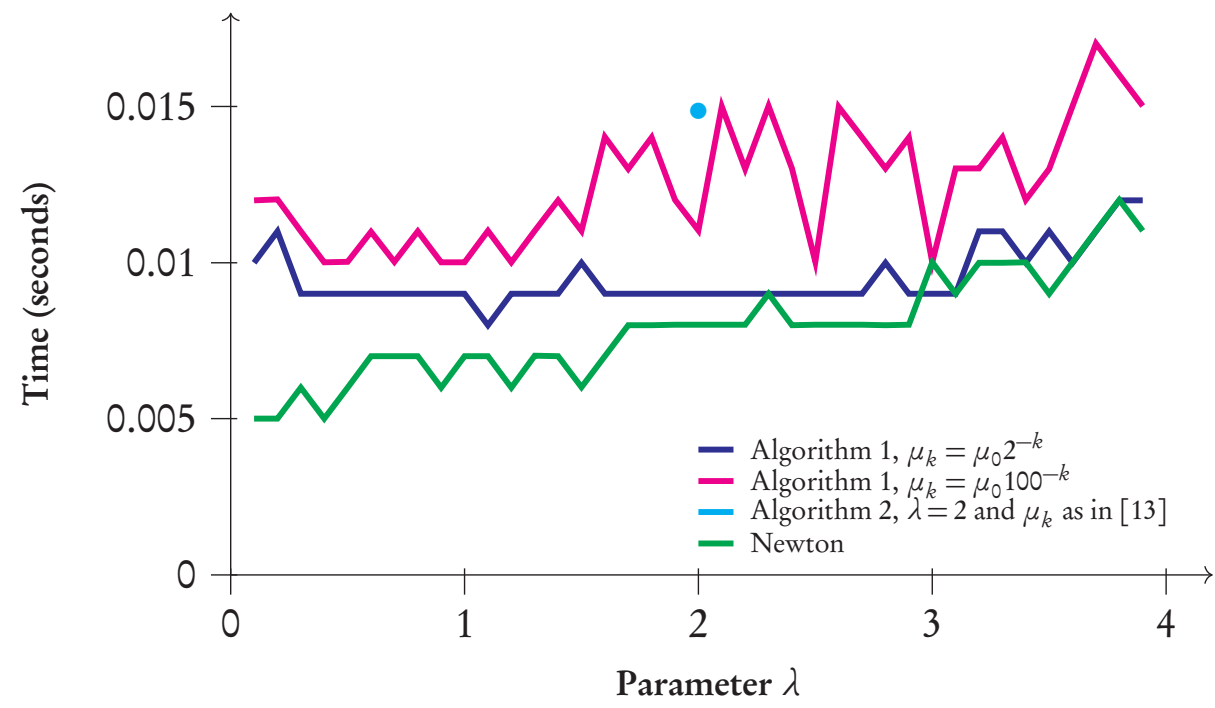

Figure 5. NCP with the function given by Example A in [24] shows another case in which the proposed algorithm is better than the other algorithms in number of iterations and computational time.

\section{Concluding remarks}

In this paper, we propose a new generalized Newton-type algorithm for solving Nonlinear Complementarity Problems based on its reformulation as a nonsmooth system of equations. To do this, we introduce a smoothing of the family of nonlinear complementarity functions presented in [7] and analyze its properties in combination with the smooth Jacobian strategy used in [17]. We prove local and quadratic convergence for the new algorithm. 
We present preliminary numerical tests that show a competitive numerical performance of the proposed algorithm compared to the traditional generalized Newton method and the Jacobian smoothing Newton method that uses the Fischer-Burmeister complementarity function, which is a particular case of the family that we consider in our proposal. These numerical tests allow us to observe that the choice of the sequence $\mu_{k}$ plays an important role in the convergence of the algorithm.

We consider that more numerical tests are needed varying the choice of $\lambda$ as well as $\left\{\mu_{k}\right\}$. Moreover, the globalization of the proposed algorithm with its global convergence analysis is also needed.

\section{Acknowledgements}

We thank the Universidad del Cauca for the time granted for this work through Research Project VRI ID 4511.

\section{Conflict of Interest}

The authors declare that they have no conflicts of interest.

\section{References}

[1] Anitescu M, Cremer JF, Potra FA. On the existence of solutions to complementarity formulations of contact problems with friction, Complementarity and Variational Problems: State of the art, SIAM Publications, 12-21, 1997.

[2] Kostreva M. Elasto-hydrodynamic lubrication: A non-linear complementarity problem, International Journal for Numerical Methods in Fluids, 4(4): 377-397, 1984.

doi: 10.1002/fld.1650040407

[3] Chen A, Oh J, Park D, Recker W. Solving the bicriteria traffic equilibrium problem with variable demand and nonlinear path costs, Applied Mathematics and Computation, 217(7): 3020-3031, 2010 .

doi: 10.1016/j.amc.2010.08.035

[4] Ferris MC, Pang JS. Engineering and economic applications of complementarity problems, SIAM Review, 39(4): 669-713, 1997.

doi: $10.1137 /$ S0036144595285963

[5] Yong L. Nonlinear complementarity problem and solution methods, Proceedings of the 2010 international conference on Artificial intelligence and computational intelligence: Part I. Springer-Verlag, 461-469, 2010. 
[6] Pang J, Qi L. Nonsmooth equations: Motivation and algorithms. SIAM Journal on Optimization, 3(3): 443-465, 1993.

doi: $10.1137 / 0803021$

[7] Kanzow C, Kleinmichel H. A new class of semismooth Newton-type methods for nonlinear complementarity problems. Computational Optimization and Applications, 11(3): 227-251, 1998. doi: 10.1023/A:1026424918464

[8] Qi L. Convergence analysis of some algorithms for solving nonsmooth equations. Mathematics of Operations Research, 18(1): 227-244, 1993.

Retrieved from

www.jstor.org/stable/3690162

[9] Broyden CG, Dennis JE, Moré JJ. On the local and superlinear convergence of quasi-Newton methods. IMA Journal of Applied Mathematics, 12: 223-245, 1973.

doi: 10.1093/imamat/12.3.223

[10] Li DH, Fukushima M. Globally convergent Broyden-like methods for semismooth equations and applications to VIP, NCP and MCP. Annals of Operations Research, 103(1): 71-97, 2001. doi: 10.1023/A:1012996232707

[11] Lopes VLR, Martínez JM, Pérez R. On the local convergence of quasi-Newton methods for nonlinear complementary problems. Applied Numerical Mathematics, 30(1): 3-22, 1999.

doi: 10.1016/S0168-9274(98)00080-4

[12] Pérez R, Lopes VLR. Recent applications and numerical implementation of quasi-newton methods for solving nonlinear systems of equations. Numerical Algorithms, 35(2), 261-285, 2004.

doi: 10.1023/B:NUMA.0000021762.83420.40

[13] Buhmiler S, Kreji'c N. A new smoothing quasi-Newton method for nonlinear complementarity problems. Journal of Computational and Applied Mathematics, 211(2): 141-155, 2008.

doi: 10.1016/j.cam.2006.11.007

[14] Dennis JE, Schnabel RB. Numerical methods for unconstrained optimization and nonlinear equations. Society for Industrial and Applied Mathematics, 1996.

doi: $10.1137 / 1.9781611971200 . f m$ 
[15] Ma C. A new smoothing quasi-Newton method for nonlinear complementarity problems. Applied Mathematics and Computation, 171(2): 807-823, 2005.

doi: 10.1016/j.amc.2005.01.088

[16] Clarke FH,Necessary Conditions for Nonsmooth Problems in Optimal Control and the Calculus of Variations, Ph.D. thesis, University of Washington, 1973.

doi: 10.1007/978-3-7643-8482-1_11

[17] Kanzow C, Pieper H. Jacobian smoothing methods for nonlinear complementarity problems. SIAM Journal on Optimization, 9(2): 342-373, 1999.

doi: $10.1137 /$ S1052623497328781

[18] Clarke FH. Optimization and nonsmooth analysis. Montreal: Society for Industrial and Applied Mathematics, 1990.

doi: $10.1137 / 1.9781611971309$

[19] Qi L.C-differentiability, C-differentialoperators and generalized Newton methods. Technical Report, School of Mathematics, The University of New South Wales, Sydney, Australia, 1996.

[20] Chen X, Qi L, Sun D. Global and superlinear convergence of the smoothing Newton method and its application to general box constrained variational inequalities. Mathematics of Computation, 67(222): 519-540, 1998.

Retrieved from

http://www.jstor.org/stable/2585135

[21] Arenas F, Martínez HJ, Pérez R. Least change secant update methods for nonlinear complementarity problem. Ingeniería $y$ Ciencia, 11(21): 11-36, 2015.

doi: 10.17230/ingciencia.11.21.1

[22] Arias CA, Martínez HJ, Pérez R. A global quasi Newton Algorithm for nonlinear complementarity problems. Pacific journal of Optimization, 13 (1): 1-15, 2017.

[23] Arenas F, Martínez HJ, Pérez, R. Redefinición de la función de complementariedad de Kanzow. Revista de Ciencias, 18(2): 111 $122,2014$.

Retrieved from

http://www.scielo.org.co/pdf/rcien/v18n2/v18n2a08.pdf 
[24] Xia Y, Leung H,Wang J. A projection neural network and its application to constrained optimization problems. IEEE Transactions on Circuits and Systems I: Fundamental Theory and Applications, 49(4): 447-458, 2002.

doi: $10.1109 / 81.995659$

\section{Un método local de suavización Jacobiana para resolver Problemas de Complementariedad No Lineal}

Resumen: En este artículo, presentamos un método de suavización para una familia de funciones de complementariedad no lineales y utilizamos sus propiedades, en combinación con la estrategia Jacobiana para el caso suave, con el propósito de introducir un nuevo algoritmo generalizado de tipo Newton para resolver un sistema no suave de ecuaciones equivalente al Problema de Complementariedad No Lineal. Además, demostramos que el algoritmo converge localmente y $q$-cuadráticamente, y analizamos su rendimiento numérico.

Palabras clave: problema de complementariedad no lineal; función de complementariedad; método de Newton generalizado; convergencia $Q$-cuadrática. 


\section{Um método local de regularização Jacobiana para resolver Problemas de Complementaridade Náo-lineares}

Resumo: Neste artigo, apresentamos um método de suavização para uma família de funções de complementaridade não lineares e utilizamos suas propriedades, em combinação com a estratégia Jacobiana para o caso suave, a fim de introduzir um novo algoritmo generalizado do tipo Newton para resolver um sistema não suave de equações equivalentes ao Problema de Complementaridade Não Linear. Além disso, demonstramos que o algoritmo converge localmente e q-quadraticamente, e analisamos seu desempenho numérico.

Palavras-chave: problema de complementaridade não linear; função de complementaridade; método de Newton generalizado; convergência $Q$-quadrática. 


\section{Favián Arenas}

Professor of the Department of Mathematics of Universidad del Cauca and member of the Optimization Research group. Degree in Mathematics (University de Sucre). Mathematics Specialist (Universidad de Córdoba, Colombia). Master of Mathematical Sciences (Universidad del Cauca, Colombia). His current research interests include systems of nonlinear equations and the nonlinear complementarity problem.

ORCID: 0000-0002-7781-7559

\section{Héctor Jairo Martínez}

Professor of the Department of Mathematics (Universidad del Valle, Colombia) and member of the ANOPI Research Group. Mathematician, surgeon and master in Basic Medical Sciences (Universidad del Valle). M.Sc. and PhD in Applied Mathematics from Rice University, Houston (USA). He has worked on research projects in the areas of optimization, linear algebra, statistics, cardiac variability and artificial neural networks, muscular physiology and mathematical epidemiology.

ORCID: 0000-0001-9747-0671

\section{Rosana Pérez}

Professor of the Department of Mathematics of Universidad del Cauca, member and coordinator of the Optimization Research Group. Degree in Mathematics (Universidad del Cauca, Colombia). Master in Mathematics (Universidad del Valle, Colombia). PhD degree in Applied Mathematics (Universidad de Campinas, Brasil). She has coordinated research projects in optimization, numerical linear algebra and neural networks, and has directed undergraduate and master's degree work.

ORCID: 0000-0003-0279-8522 